\title{
Child behaviour after anaesthesia: association of socioeconomic factors and child behaviour checklist to the post-hospital behaviour questionnaire
}

\author{
M. Karling (mats.karling@vll.se) ${ }^{1}$, B. Hägglöf ${ }^{2}$ \\ 1.Divisions of Anaesthesia and Child and Adolescent Psychiatry, University Hospital of Northern Sweden, University of Umeå, Umeå, Sweden \\ 2.Department of Clinical Science, Division of Child and Adolescent Psychiatry, University Hospital of Northern Sweden, SE-901 85 Umeå, Sweden
}

\section{Keywords}

Anaesthesia, Child, Child behaviour, Demographic factors, Hospitalization, Social class

\section{Correspondence}

Mats Karling, MD, Department of Anaesthesia and Intensive Care, University Hospital of Northern

Sweden, SE-901 85 Umeå, Sweden.

Tel: +46-90-7850000 | Fax: +46-90-136235 |

Email: mats.karling@vll.se

\section{Received}

13 April 2006; revised 30 August 2006; accepted 1 September 2006.

DOI:10.1111/j.1651-2227.2007.00108.x

\begin{abstract}
Aim: In the process of validation of the Swedish translation of the Post-Hospital Behaviour Questionnaire (PHBQ) to assess its relation to the Child Behaviour Checklist $(\mathrm{CBCL})$ and to describe its relation to sociodemographic factors.

Method: Three hundred and forty children 2-13 years studied in connection with elective procedures which included anaesthesia. Parents completed the CBCL forms before and after hospitalization as well as the PHBQ 2 weeks after hospitalization.

Results: In multivariate analysis of PHBQ total score independent risk factors were: age $<5$ (OR 2.4; $\mathrm{Cl} 1.4-4.0)$, living in a one parent family (OR 4.4; $\mathrm{Cl}$ : 1.6-12.6) and not living in a rural area (OR 1.6; $\mathrm{Cl}$ : 1.0-2.6). The correlation between the total scores for PHBQ and CBCL for children aged 2-4 was moderate: $(r=0.38 ; p<0.005)$. For children aged $4-7$ there were significant correlations between PHBQ sleep anxiety and CBCL Other problems $(r=0.4 ; \mathrm{P}<0.01)$, PHBQ eating disturbances and $C B C L$ total score $(r=0.3 ; P<0.01)$, though none of these significant correlations were observed for children above 7 years of age.

Conclusion: There is an association between PHBQ and CBCL which is weaker for older children. The reason for this might be that $\mathrm{PHBQ}$ is more sensitive in the younger age group and to minor changes in behaviour. Children younger than 5 years of age or living in a one parent family or not living in rural areas appear to have higher incidence of problematic behaviour in a 2 week follow up after anaesthesia.
\end{abstract}

For many years it has been known that children may react to hospitalization with behavioural problems (1). Behaviours like nightmares, eating problems, separation anxiety and regression have been described (2). The Post-Hospital Behaviour Questionnaire (PHBQ) (3), the most widely used questionnaire to measure behavioural change after hospitalization of children, was developed and validated in 1966. The factor structure, face validity and reliability has recently been investigated in a Swedish translation of the questionnaire (4), and the results demonstrated a factor structure which was somewhat different from the original one. This study is the next step in the validation process, aimed to investigate its construct validity in a Swedish translation and population. The goal is to make the PHBQ a useful tool for further studies in the psychological impact of hospital care of children.

The construct validity of the PHBQ have been tested by comparing the total score from the questionnaire with ratings done by a child psychiatrist who interviewed 20 children 1 week after tonsillectomies $(r=0.45 ; \mathrm{p}<0.05)$ (3). The ratings were confirmed by a child psychologist who analysed the interview from tape recordings. The influence of social and occupational status was roughly measured by comparing type of hospitalization (clinic, multiple-bed and private). The PHBQ designers reported that children from the clinic seemed to improve their behaviour in one factor (General anxiety and regression; $\mathrm{p}<0.001$ ) after hospital- ization, the others were unchanged in the validation study (3). To our knowledge there has been no other construct validation and investigation of the relation to demographic data of the PHBQ since its original presentation.

In the construct validation process The Child Behaviour Checklist (CBCL) $(5,6)$ was chosen as standard as it is a widely used instrument to asses emotional and behavioural problems in children and one the very few validated in the Swedish language (7).

We hypothesized the relation of sociodemographic data to behavioural change after hospitalization and anaesthesia indicated by the PHBQ could be confirmed with the CBCL and the behavioural changes indicated by the PHBQ could be verified with the CBCL.

\footnotetext{
Design

Children, 2-13 (mean $7.0 \pm 2.9$ ) years of age, which were scheduled for an elective procedure that included anaesthesia, either because of surgery or for diagnostic purposes, were consecutively recruited. The exclusion criteria were oncological disease, mental retardation or that parents could not speak or understand Swedish. All children except 14 were treated in the University Hospital of Northern Sweden, Umeå. The rest were from Queen Silvia Child and Youth Hospital in Gothenburg, Sweden.
} 
Two weeks before admittance, the parents were asked to complete a questionnaire concerning demographic data, number of earlier hospitalizations and anaesthesias, and the parents of the children from Umea were asked to complete the Swedish version of the CBCL (CBCL/2-3 (6) or CBCL/4-18 (5). A short written form, including information about the hospital procedure, was sent to the parents at the same time.

Two weeks after returning home, the PHBQ and a second CBCL (only children from Umeå) questionnaire were sent to the parents. If not answered within 1 week, a reminder was sent.

The Regional Ethic Review Board at Umeå and Gothenburg Universities approved the study.

\section{PATIENTS AND METHODS}

The study population is the same as in our previous study of the PHBQ (4). Data were collected during 2002 and 2003. There were 614 children eligible for the study, but 169 declined to participate, with a further 71 lost to follow up by administrative reasons (lost questionnaire). The remaining 375 received follow up questionnaires, and of those, 35 never were returned. This means that 340 participated fully in the study, and the response frequency was 55\%. CBCL was only distributed to the families in Umeå, which means that 326 was possible for CBCL calculations, 10 of those did not return the CBCL Questionnaire and further 14 of those had to many missing items $(>8)$. The response rate for CBCL was thus $50 \%$. There were no difference in age or gender between dropouts and participants. Mean age for dropouts were 6.78 and for the participants 6.97 ( $\mathrm{p}=0.45, t$-test). There were $59 \%$ boys, $41 \%$ girls among the participants and $65 \%$ boys, $35 \%$ girls among the dropouts $(\mathrm{p}=0.18$, Chi-square test).

\section{The PHBQ}

The PHBQ consists of 25 items. Parents are asked to compare any current behavioural problems of their child with the period before hospitalization. Five alternatives are provided: $1=$ much less, $2=$ less, $3=$ unchanged, $4=$ more and $5=$ much more. The total score was computed by adding the individual item scores. Five subscales are provided: General anxiety-withdrawal, Eating disturbances, separation anxiety, Regression-aggression and Sleep anxiety (4). When analysing the relation to demographic data the PHBQ was dichotomized: no change or less problematic behaviours, or more. The PHBQ exists in an absolute form where the parents fill out the questionnaire both before and after hospitalization, but the relative form is supposed to be more sensitive while it may be more subjective (2).

\section{The CBCL}

The CBCL consists of two parts, but in this study only the items from behavioural/emotional portion was used. This consists of 100 items for children between 2 and 4 years of age (CBCL/2-3) and 113 for children from 4 years of age and older (CBCL/4-18). The parents are asked to answer the items in a three point scale: $0=$ do no agree, $1=$ agrees somewhat or sometimes, 2 = fully agree. The CBCL behavioural/emotional portion is divided into two broad-band dimensions: Internalizing and Externalizing. The Internalizing scale consists of three subscales in the form for ages 4 to 18: Withdrawn, Somatic complaints and Anxious-depressed, while in the 2-3 form the Somatic complaint subscale does not exist. The Externalizing scale consists of Delinquent behaviour (age 4-18), Destructive behaviour (age 2-3) and Aggressive behaviour. The total score is computed by the sum of all subscales excluding item 103, which is omitted in the 4-18 version.

The younger group consisted of 53 children and the older 249. To estimate any change in behaviour with the CBCL, the differences in item scores between after and before hospitalization were calculated.

\section{Statistics}

The correlation between CBCL scores and PHBQ were determined by Spearman rank correlation. Univariate and multivariate analyse between demographic data and PHBQ scores were analysed by using binary logistic regression where the PHBQ scores were dichotomized: no change or less problematic behaviours, and more problematic behaviours. Comparisons between groups were done by Mann-Whitney test. All statistical analysis was made by using SPSS 11.5.

\section{RESULTS}

\section{Relations between the PHBQ and demographic data}

Demographic data are presented in Table S1 (in Supplementary material online). Univariate data are presented in Table S2 (in Supplementary material online). Age, domicile not in rural areas and living in a one adult household had significant associations to a PHBQ score indicating increasing problematic behaviour. Multiple logistic regression was performed by including all variables and stepwise excluding the most insignificant variable one by one. Age less than 5 years and being a child in a one adult household, was associated to an increased PHBQ total score (Table S3, in Supplementary material online). Age less than 5 years was a significant predictor to increased problematic behaviour in all subscales except eating disturbances. Living in a one adult household was associated with high scores in Separation anxiety, Regression-aggression and Sleep anxiety. Not living in rural areas was associated with Eating disturbances and Separation anxiety. Separation anxiety was also associated to mothers with education lower than university and living in a family with more than two children.

\section{Relations between PHBQ and CBCl}

For children less than 4 years of age there was a significant correlation between the totals scores for the CBCL difference and the total PHBQ scores (Table S4, in Supplementary material online). The total PHBQ score was furthermore significantly correlated to internalizing, Aggressive behaviour and Anxious-depressed scales in the CBCL. Sleep anxiety in 
the PHBQ was also significantly correlated to these scales and to Externalizing and Somatic problems in the CBCL.

For children between 4 and 7 years of age there were significant correlations between subscales in the PHBQ Sleep anxiety and the CBCL other problems, PHBQ Eating disturbances and CBCL total score (Table S5, in Supplementary material online). Eating disturbances in the PHBQ had also significant but weak correlations to Anxious depressed, attention problems, Aggressive behaviour, Other problems and Externalizing in the CBCL.

For children from 7 years and older there were no correlations except a weak negative correlation between thought problems in CBCL and aggression-regression in PHBQ.

To assess if the findings of the relation between PHBQ and demographic data could be confirmed with the CBCL, the demographic groups which correlated to the PHBQ subscales in the multivariate analyse were compared in their CBCL scoring. Only children 4 years of age and older were included in this analyse since there were too few patients in the lower age group. Children from 4 to 7 years of age had higher CBCL subscale scores differences (mean -0.34 \pm 1.43 ) than older children (mean $-0.80 \pm 2.00 ; \mathrm{p}=0.02$ ) for Anxious-depressed indicating less improvements. Children from families with less than 3 children had lower mean CBCL subscale scores differences for social problems $(-0.42$ $\pm 1.20)$ and Attention problems $(-0.75 \pm 1.60)$ than children from larger families (social problems $-0.06 \pm 0.0 .95 ; \mathrm{p}=$ 0.05 , Attention problems $-0.028 \pm 1.17 ; \mathrm{p}=0.003$ ). Social problems in families with mothers with university education seemed to improve less (mean $-0.16 \pm 1.18$ ) than children from families with lower education (mean $-0.43 \pm 1.04$; $\mathrm{p}=0.03$ ).

For children living in one adult household, compared with other children, there was no difference before hospitalization in any CBCL subscale, but after hospitalization they had higher mean CBCL scores for Social problems $(1.53 \pm 1.64$ vs. $0.87 \pm 1.32 ; \mathrm{p}=0.02)$ and Somatic complaints $(1.47 \pm$ 1.13 vs. $0.96 \pm 1.44 ; \mathrm{p}=0.05)$. Children living in rural areas seemed to have less Somatic complaints $(1.06 \pm 1.68)$ than children from larger villages $(1.47 \pm 1.71 ; \mathrm{p}=0.02)$ before hospitalization. After hospitalization there was no difference $(0.83 \pm 1.31$ vs. $0.97 \pm 1.38 ; \mathrm{p}=0.5)$.

These findings in the CBCL were consistent for children less than 7 years old, living in a one parent household and living in a non rural was associated with higher PHBQ scores, indicating more problematic behaviour. There was no indication that mothers with non-university education were a risk factor for problematic behaviour outcome measured with the PHBQ.

By dichotomizing the populations in the two age groups by PHBQ total score, less than or equal to 76 , indicating no increasing behavioural problems and more than 76 , indicating net onset of behavioural problems (no overall change is 75), changes in CBCL were studied (Fig. 1).

Children, ages 4 and above, with total PHBQ score above $76(\mathrm{~N}=62)$, had higher total CBCL scores both before and after hospitalization compared with those with lower PHBQ scores $(\mathrm{N}=185)$ (CBCL before hospitalization and PHBQ $\leq 76$ median 15 (quartiles: 8.0; 24.0), PHBQ $>76$ median $20.5(11.75 ; 31.75) \mathrm{p}=0.008$. CBCL after hospitalization and PHBQ $\leq 7610$ (5.0; 19.0), PHBQ > $7616.0(8.75 ; 23.75)$; $\mathrm{p}=0.000)$.

Children, ages 2-4 years, and PHBQ score above 76 $(\mathrm{N}=16)$ had similar changes (CBCL before hospitalization and PHBQ $\leq 76(\mathrm{~N}=37)$ median19.0 $(11.0 ; 41.5)$ and 10.5 $(4.0 ; 32.5)$ after, PHBQ > 76 median $24.5(16.25 ; 33.25)$ before and $27.5(7.5 ; 41.25)$ after, NS) but only the difference in CBCL between before and after was significant (PHBQ $\leq 76$ median $-6.0(-11.0 ;-1.0)$ PHBQ $>76$ median -0.5 $(-7.25 ; 10.5) ; \mathrm{p}=0.02)$.

\section{DISCUSSION}

In this study, the impact of demographic factors on the PHBQ score and the relation to another behaviour measurement instrument, the Child Behaviour Checklist, was investigated. Children less than 5 years of age as well as children in families with only mother as caretaker and living in rural areas seemed to be independent risk factors for more problematic behaviour after hospitalization. There were significant but not strong correlations between the CBCL and PHBQ scores in children less than 7 years of age. In older children no significant correlation was found. The findings of the relations between sociodemographic data and PHBQ scores could in most cases be replicated by analysing CBCL scores before and after hospitalization.

The dropout frequency in this study was quite high; the response rate was $55 \%$. If the 71 questionnaires, which were lost due to administrative reasons were not included, since this is probably a random process not introducing bias, the response rate would have been 63\% for the PHBQ and 58\% for the CBCL. The response rate in the original study was $48 \%$ (3).

\section{Age}

Many authors have found that younger children are more prone to develop problematic behaviours than the older children, measured by the PHBQ (8-12). The results of this study are similar to those findings and there is evidence to support this by the use of the CBCL. Many of the items in the PHBQ reflect behaviours in younger children (e.g. Does your child need a pacifier? Does your child suck her fingers or thumbs? Does your child seem to get upset when you leave him or her for a few minutes?) It is possible that older children also react to hospitalization in a negative way but this reaction is not detected by the PHBQ.

\section{Family structure}

Children living in a one-parent-family are at risk for development of psychosocial problems $(13,14)$ and have an increased risk for psychiatric disease (15). In children with asthma parent reported poorer physical health, mental health and social functioning in one parent families compared with children in two parent families (16). This may be explained in part by worse economic status. In this study there were no significant differences in CBCL score between children from one 


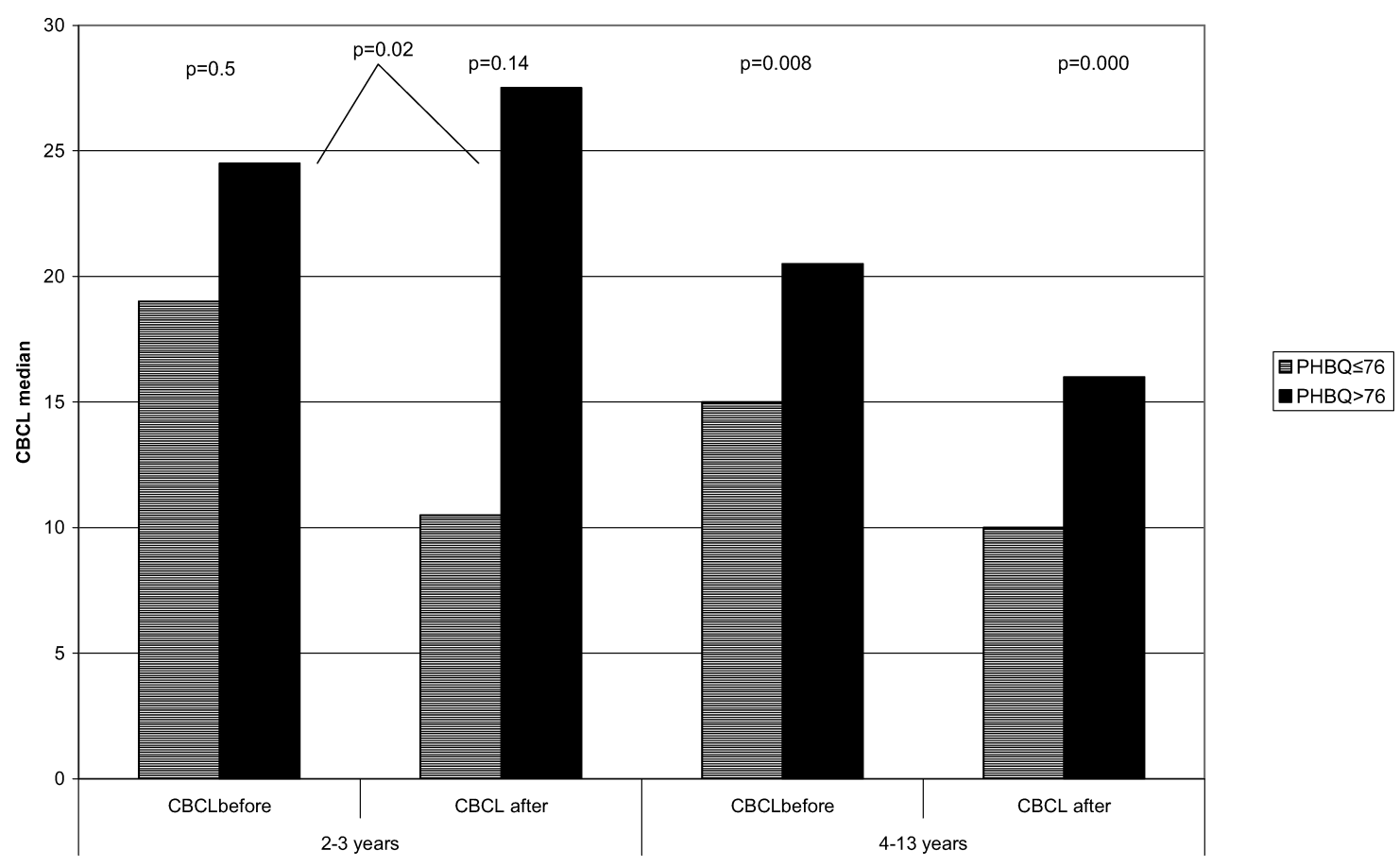

Figure $1 \mathrm{CBCL}$ scores before and after hospitalization in relation to PHBQ groups with no problematic behaviour (PHBQ total score $\leq 76$ ) and to deterioration in behaviour (PHBQ total score $>76)$. CBCL after and before difference is significantly $(P=0.02)$ different between the two PHBQ groups in the younger age group.

parent families other children before hospitalization, though regardless of other socioeconomic factors they have an increased risk for deterioration in their behaviour measured both by PHBQ and CBCL, at least in a short term follow up.

\section{Area of residence}

The PHBQ results indicate that children living in rural areas seem to have less risk for development of problematic behaviour after hospitalization. In an epidemiologic study of Swedish children (7), children living in rural areas had lower scores in the CBCL. We found similar results when examining behaviour before hospitalization, but no difference between groups was found after hospitalization.

\section{Parental education}

Lower parental education was related to the higher scores in the CBCL (17). Our results for the PHBQ showed that only the education of the mother and not the father is associated with the outcome. The CBCL scores indicated that children to mothers with lower education seem to improve more after hospitalization. Since socioeconomic factors are related to CBCL scores and to parental education (7) and since the education of the father had no influence, this finding is likely due to maternal education and not other socioeconomic factors.

In this study the difference between two CBCL test results collected in series were calculated. With this study design a 'practice effect' or attenuation in the second results, due to repeated exposure to the same questionnaire, may be intro- duced. The size of this attenuation has been estimated to be between 0.84 to 0.94 (18) for a mean time of 8 days. It is recommended that the time interval between test and retest should be a least 1 month (18). Since the course of this study was approximately 3-4 weeks, it is difficult to estimate the size of the attenuation, but it would tend to diminish the difference and probably the correlation between PHBQ and CBCL. In our case, the mean difference between after and before hospitalization was mainly negative, indicating less problematic behaviours after hospitalization, but this may in fact not be valid if the second CBCL scoring was confounded significantly by 'practice factor'.

\section{Correlations between PHBQ and CBCL}

For the lower ages the there were significant but not high correlations between CBCL/2-3 and PHBQ total scores. Sleep anxiety in the PHBQ correlated to several of the subscales of CBCL but not on Sleep problems. These findings demonstrate an association between the two scales. For children between 4 and 7 correlations between PHBQ Eating disturbances were found to be strongest to the CBCL total score and also to Other problems which has questions about eating. The PHBQ Sleep anxiety correlated best with the CBCL Other problems, which contains questions about sleep.

It appears that a higher score for a child in CBCL prior to hospitalization is associated with risk for more problematic behaviour measured by PHBQ, at least for older children. For younger children the tendency is the same, but the number of children in this study is not enough to confirm this. 
The weak associations between the two scales could be explained by several factors.

There could be attenuation in the second CBCL scoring due to the practicing effect which would tend to lower the scores after the hospital visit. Secondly, the fact that the parent is asked to estimate the change in behaviour after hospitalization might be a more sensitive measure rather than two different measures for the behaviour as they see their child when scoring. Thirdly, it is not certain that the two instruments measure the same thing. The CBCL is primarily focused on psychiatric or general behavioural problems that might be correlated to psychiatric, social or neurological abnormalities and may only reflect greater behavioural problems while not catching less problematic behaviours. The PHBQ is more focused on changes that occur after a stressful event. The items in the PHBQ may reflect more subtle changes in behaviour.

\section{CONCLUSION}

Young age, living in a one adult household and living in a town was associated to higher scores, indicating development of problematic behaviours after hospitalization including anaesthesia, if measured by the PHBQ. There is support for these findings with a concurrent use of the CBCL. There is an association between the PHBQ and the CBCL at least for younger children and it seems that the PHBQ is more sensitive to problematic behaviours in this population. Further research is warranted for the construct validity of the PHBQ in a Swedish population. Whether or not older children are less affected by hospitalization, or if the items in the PHBQ are not reflecting their behaviour changes, is still not clear. Further research is needed to find ways that make hospitalization less stressful to younger children.

\section{ACKNOWLEDGEMENTS}

This study was supported by a grant from Oscarsfonden and Insamlingsfonden from the University of Northern Sweden. The authors will thank Karin Lundbäck och Minette Wilén for their work with data collection. To Hans Stenlund for his assistance with the statistics and to Michael Haney for his help with the English language.

\section{References}

1. Zetterstrom R. Responses of children to hospitalization. Acta Paediatr Scand 1984 May; 73(3): 289-95.

2. Thompson RH, Vernon DT. Research on children's behavior after hospitalization: a review and synthesis. J Dev Behav Pediatr 1993 Feb; 14: 28-35.

3. Vernon DT, Schulman JL, Foley JM. Changes in children's behavior after hospitalization. Some dimensions of response and their correlates. Am J Dis Child 1966 Jun; 111: 581-93.

4. Karling M SH, Hägglöf B. Behavioural changes after anaesthesia: validity and liability of the post hospitalisation behaviour questionnaire in a swedish paediatric population. Acta Paediatr 2005; 95: 340-6.

5. Achenbach T. Integrative guide for the $1991 \mathrm{CBCL} / 4-18$,YSR and TRF profiles. Burlington, VT: University of Vermont, Department of Psychiatry, 1991.
6. Achenbach T. Manual for the Child Behaviour Checklist/2-3 and 1992 Profile. Burlington, VT: University of Vermont, Department of Psychiatry, 1992.

7. Larsson B, Frisk M. Social competence and emotional/behaviour problems in 6-16 year-old Swedish school children. Eur Child Adolesc Psychiatry 1999 Mar; 8: 24-33.

8. Kotiniemi LH, Ryhanen PT, Moilanen IK. Behavioural changes following routine ENT operations in two-to-ten-year-old children. Paediatr Anaesth 1996; 6: 45-9.

9. Kotiniemi LH, Ryhanen PT, Moilanen IK. Behavioural changes in children following day-case surgery: a 4-week follow-up of 551 children. Anaesthesia 1997 Oct; 52: 970-6.

10. Kain ZN, Caldwell-Andrews AA, Maranets I, McClain B, Gaal $\mathrm{D}$, Mayes LC, et al. Preoperative anxiety and emergence delirium and postoperative maladaptive behaviors. Anesth Analg 2004 Dec; 99: 1648-54, table of contents.

11. Kain ZN, Mayes LC, O'Connor TZ, Cicchetti DV. Preoperative anxiety in children. Predictors and outcomes. Arch Pediatr Adolesc Med 1996 Dec; 150: 1238-45.

12. Visintainer MA, Wolfer JA. Psychological preparation for surgery pediatric patients: the effects on children's and parents' stress responses and adjustment. Pediatrics 1975 Aug; 56: 187-202.

13. Jellinek M, Little M, Murphy JM, Pagano M. The Pediatric Symptom Checklist. Support for a role in a managed care environment. Arch Pediatr Adolesc Med 1995 Jul; 149: 740-6.

14. Jellinek MS, Murphy JM, Little M, Pagano ME, Comer DM, Kelleher KJ. Use of the Pediatric Symptom Checklist to screen for psychosocial problems in pediatric primary care: a national feasibility study. Arch Pediatr Adolesc Med 1999 Mar; 153: 254-60.

15. Weitoft GR, Hjern A, Haglund B, Rosen M. Mortality, severe morbidity, and injury in children living with single parents in Sweden: a population-based study. Lancet 2003 Jan 25; 361: 289-95.

16. Sawyer MG, Spurrier N, Whaites L, Kennedy D, Martin AJ, Baghurst P. The relationship between asthma severity, family functioning and the health-related quality of life of children with asthma. Qual Life Res 2000; 9: 1105-15.

17. Bohlin G, Janols LO. Behavioural problems and psychiatric symptoms in 5-13 year-old Swedish children-a comparison of parent ratings on the FTF (Five to Fifteen) with the ratings on CBCL (Child Behavior Checklist). Eur Child Adolesc Psychiatry 2004; 13(Suppl 3): 14-22.

18. Thomas MA, Leslie a, Rescorla. Manual for the ASEBA Schoolage forms and Profiles: Libary of Congress, 2001.

\section{Supplementary material}

The following supplementary material is available for this article:

Table S1 Demographic data.

Table S2 Odds Ratios (OR) and confidence intervals (CI) for PHBQ for association between demographic data and risk of negative behaviour outcome.

Table S3 Odds ratios (OR) and confidence intervals (CI) for PHBQ for the association between demographic data and risk of negative behaviour outcome.

Table S4 Correlation between PHBQ and difference between CBCL/2-3 before and after hospitalization.

Table S5 Correlation between CBCL/4-18and PHBQ, children between 4 and 7 years and factors $\mathrm{N}=94$. 
This material is available as part of the online article from: http://www.blackwell-synergy.com/doi/abs/10.1111/j.16512227.2007.00108.x

(This link will take you to the article abstract).
Please note: Blackwell Publishing are not responsible for the content or functionality of any supplementary materials supplied by the authors. Any queries (other than missing material) should be directed to the corresponding author for the article. 